\title{
CLOCKING IN TURBINES: REMARKS ON PHYSICAL NATURE AND GEOMETRIC REQUIREMENTS
}

Jerzy Swirydczuk, Assoc. Prof. Institute of Fluid-Flow Machinery

Polish Academy of Science, Poland

\begin{abstract}
The article discusses two issues relating to the clocking phenomenon in turbines, which are the physical course of stator wake deformation in rotor passages and its further interaction with downstream stator blades, and turbine geometry parameters which are believed to be most favourable for clocking. In both cases, the results presented in the article have made it possible to verify and reformulate the previously accepted opinions.
\end{abstract}

Keywords: turbines, clocking, wakes, vortices, blade counts

\section{Introduction}

Clocking, also sometimes referred to as indexing, consists in changing the circumferential position of one stator (or rotor) row by rotating it with respect to the other stator (rotor) in a multistage fluid-flow machine. Provided that certain geometric conditions are met, it can be realised in all types of fluid-flow machines, including those used in marine industry. A basic motivation for investigating and applying clocking in turbines was an attempt to control the wake interaction in the clocked rows and this way to improve turbine performance. Flow effects generated by clocking were the object of examination of numerous researchers. Huber et al. investigated them experimentally on a twostage turbine with blade counts in consecutive rows equal to 54:50:54:50 [1]. Their experiments revealed that about $0.8 \%$ of efficiency gain can be obtained due to clocking. For the same turbine, a numerical analysis for the two-dimensional midspan geometry was performed by Griffin et al. [2], who correctly predicted the clocking position corresponding to the maximal efficiency, although the predicted efficiency gain was only equal to $0.5 \%$, i.e. remarkably less than that obtained in the experiment. Clocking effects in a 1-1/2 stage turbine have been numerically simulated by Eulitz et al. [3] and Dorney and Sharma [4] who concluded that larger unsteady pressure amplitudes on the blades correspond to higher-efficiency configurations. This conclusion was later confirmed by Cizmas and Dorney [5], although an opposite trend was also reported by Griffin [2] and Dorney et al. [6]. Reinmoller et al.[7] studied experimentally and numerically clocking related phenomena in a 1.5 stage axial turbine. Relative efficiency changes in their experiment amounted to $1.0 \%$, against $0.7 \%$ in the corresponding calculations.

The effect of airfoil clocking on a six-row turbine has been also investigated by Cizmas and Dorney [8]. A comprehensive analysis of the clocking phenomenon presented in that paper included rotor clocking, clocking of multiple rows, and the concept of full turbine clocking, including all rotor and stator rows. For the examined turbine the second-stage clocking produced larger efficiency gains than the third-stage clocking, which was true for both rotor and stator. The results presented in the article also revealed that rotor clocking can be a source of efficiency gains being twice as large as those produced by stator clocking.

Li Wei et al examined numerically clocking effects in a 1.5 stage axial low-pressure turbine in which the blade count ratio in the clocked stators was $\mathrm{n}_{\mathrm{S} 1}: \mathrm{n}_{\mathrm{S} 2}=84: 126=2: 3$, obtaining only $0.1 \%$ efficiency gain [9].

The present article discusses two opinions on clocking, which have been formulated based on the knowledge gained in experimental investigations and numerical studies of the phenomenon. The first of them refers to the physical nature of wake/blade interactions in the clocked rows and says that the greatest benefits of clocking are achieved when the wake of the upstream stator impinges on the downstream stator leading edge, while an efficiency drop is observed when the wake is convected through the middle of the downstream stator passage $[2,3,4,7]$. The second opinion refers to 
geometric requirements for the clocked rows and says that larger efficiency benefits can be obtained when the blade count ratio of the clocked rows is close to one, and when it is far from unity the clocking brings practically no effect. This opinion can be found in articles by Arnone et al. [10-12]. Also other authors apply a procedure which scales the geometry of the examined turbine stages with slightly differing blade counts to obtain the blade count ratio exactly equal to one $[6,8]$.

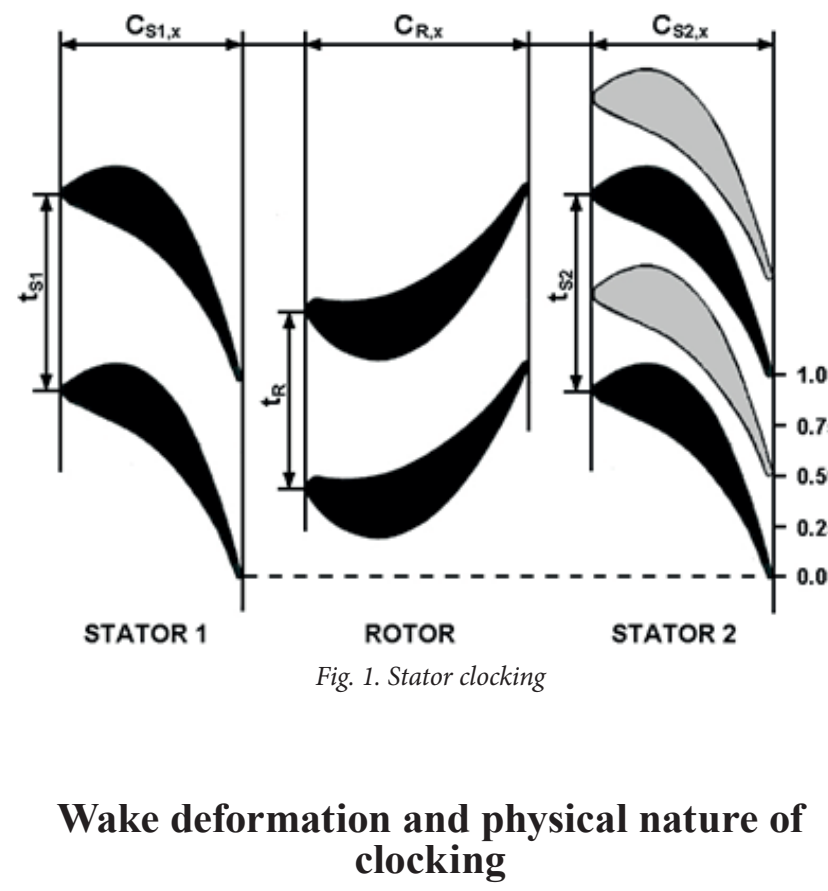

As mentioned in the Introduction, clocking in a multistage fluid-flow machine consists in rotating one stator/rotor row with respect to the other. Fig. 1 explains technical realisation of stator clocking in a turbine by presenting two clocking positions of the second stator. Numbers on the right represent the values of a parameter assumed as the measure of clocking which expresses the ratio of the current rotation angle $\alpha$ to its full clocking range $\alpha_{\text {clock }}$, which in classical clocked rows with the same blade counts: $\mathrm{n}_{\mathrm{s} 1}=\mathrm{n}_{\mathrm{s} 2}$, is equivalent to the rotation by the angle $\alpha_{t}$ corresponding to one blade pitch. The basic configuration, labelled by the clocking parameter assumed as 0.0 , is where the trailing (or leading) edges of the clocked stators $S_{1}$ and $S_{2}$ are situated along the same line parallel to the turbine axis, the dashed line in Fig. 1 . The second position (gray profiles) is obtained by rotating circumferentially stator $\mathrm{S}_{2}$ by an angle $0.5^{\star}$ at corresponding to half of the stator pitch.

All clocking effects generated in stator $\mathrm{S}_{2}$ are believed to be the result of its interaction with the wakes shed from the trailing edges of blades composing the $\mathrm{S}_{1}$ cascade, therefore the starting point for the present analysis of the physical nature of clocking will be the analysis of deformation experienced by $S_{1}$ wakes on their way through rotor $\mathrm{R}$ passages. Throughout the history, this process was modelled and interpreted in various ways depending on current knowledge on the inner structure and behaviour of stator wakes. In one of first wake models, proposed by Meyer [13], the wake was simply represented by a so-called "negative jet", being a regular area of velocity disturbances in the otherwise uniform flow. The stator wakes, convected with the main flow, were chopped into smaller parts by downstream rotor blades, modelled as straight line segments. While flowing through the rotor, the wake segments preserved their initial shapes and behaved as negative jets generating secondary velocity field which transported the fluid from the pressure side to the suction side of the rotor passage.

Then, attempts were made to include the ability of the wake to deform on its way through the rotor passage. Smith [14] observed that the circulation around the rotor blade makes the opposite ends of the wake segment move with different velocities. As a consequence, the segment is stretched and sheared in the rotor passage, and when the wake leaves the rotor it forms a saw-like shape rather than preserves the initial continuous structure. The first attempt to capture wake deformation was made by Lienhart [15] who modelled the wake using the potential flow with a sequence of isolated vortices generated in the vicinity of blade trailing edges. Once generated, the vortices moved with the local flow velocity through the rotor, thus contributing to unsteady flow effects inside the rotor passages. Successive wake deformation stages were numerically simulated, and unsteady fluctuations of potential forces generated in each row were calculated. This model was modified by Krammer [16] who extended the vortex model of the wake by adding two contra-rotating rows of vortices along the wake axis to simulate the velocity defect. Thus this wake model, consisting of two types of vortices, was able to combine potential and viscous effects. He presented the course of wake deformation individually for viscous and potential parts, and calculated their impact on unsteady forces generated on the rotor. It is noteworthy that despite a discrete representation, the wakes in those papers were still treated in a classical manner, as continuous bands of fluid in otherwise inviscid and incompressible flow, with the velocity deficit changing uniformly along the wake axis.

Much more accurate modelling of the stator wake and its interaction with the rotor cascade was possible thanks to the development of specialised 3D codes to solve Reynolds Averaged Navier-Stokes (RANS) equations for turbulent, compressible, and viscous flows in fluid-flow machines. In those codes the stator wakes were obtained as part of the solution and their characteristics did not have to be assumed a priori. Experimental verification of the obtained results was, as a rule, good.

Early RANS calculations made use of relatively coarse grids and produced wakes which had the form of regular bands of higher entropy, without visible traces of inner structure. Estimating clocking gains from these calculations has lead to a commonly accepted conclusion, mentioned in the Introduction, that the best clocking configuration is when the $S_{1}$ wake impinges on the leading edges of $S_{2}$ blades while passing of the wakes through the middle of the $S_{2}$ passages results in efficiency drop. Fig. 2 illustrates these two most and least favourable cases by presenting instantaneous patterns of unsteady entropy distributions in the passages of the both clocked stators and the rotor moving between them. Different stator/rotor blade count ratio provides an opportunity to observe consecutive stages of the $S_{1}$ wake motion through the $\mathrm{S}_{2}$ cascade. 

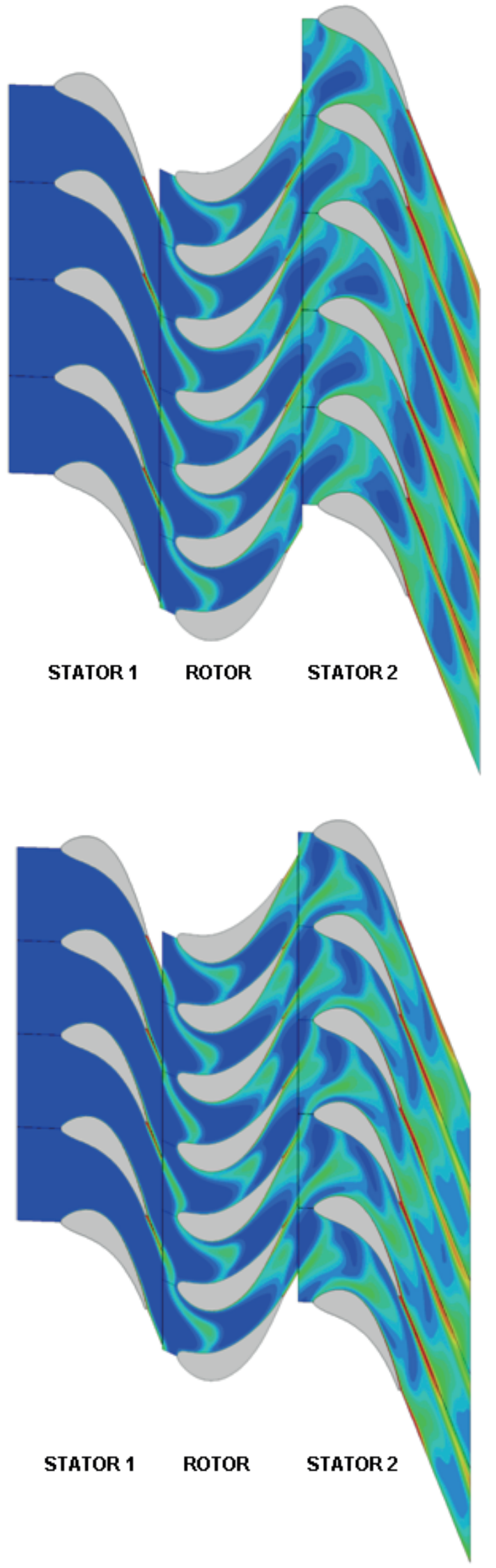

Fig. 2. Stator clocking configurations: advantageous (up) and disadvantageous (down)
More recent experiments oriented on taking more detailed insight into the inner structure of stator wakes revealed characteristic frequencies in the flow signals recorded in the wake, which testified to the presence of regular minor structures in the wake area $[17,18]$. Also numerical computations performed for isolated stators at sufficiently fine grids and time steps allowed to produce wake patterns revealing two rows of well developed vortices having the structure similar to that of the von Karman vortex street, [19-22].

Some initial attempts to take into account vortex dynamics when analysing the stator wake motion through the rotor cascade have been published. A most detailed study was done by Kost et al. [23], Tiedemann and Kost [24] and Hummel [25], who examined experimentally and numerically unsteady flow fields generated by stator wakes in rotor passages of a full size aero-engine high-pressure turbine stage having 43 stator blades and 64 rotor blades. Visual studies of the flow inside the rotor passages performed by Kost et al. with the aid of coolant injected to the flow at the vicinity of stator blade trailing edges have revealed that on its way through the rotor passage the stator wake forms two separate areas of different-sign vorticity which move one after the other through the passage, thus creating the earlier mentioned negative jet between the blade walls, clearly observed on the numerically calculated distributions of velocity vectors.

In their experimental study, Tiedemann and Kost focused on final effects of the deformation of stator wakes in the rotor passage and their further interaction with the rotor wakes. They recorded velocity and pressure fluctuations at the rotor passage exit for different positions of stator clocking with respect to the positions of the measuring sensors. Based on the obtained results they concluded that the impingement of the S1 wake on the leading edge of a hypothetical stator S2 would decrease the fluctuations, which may affect the development of the stator's boundary layer and the resultant losses. They also observed considerable reduction in flow incidence angle variations caused by the passing stator wakes which, according to their opinion, would also contribute to the benefits of clocking.

The stator/rotor wake interaction in the same turbine has been examined numerically by Hummel. In his calculations the structure of the stator wake observed downstream of the rotor also revealed two well separated areas of positive and negative vorticity which behaved in the same way as that experimentally observed by Kost et al. One of conclusions from his study was that stator vortices, when interacting with the rotor wakes on its both side, increase the strength or rotor vortices when the velocity vectors generated by the stator vortex and the rotor vortex coincide with each other in the area between them, and decreases when these vectors are opposite to each other.

The process of stator wake deformation in rotor passages was also studied by Swirydczuk $[26,27]$ who used for this purpose a model of potential flow with vortex singularities representing stator and rotor wakes. Figure 3 shows an instantaneous pattern of stator and rotor wake vortices. The form of the stator wake in front of the rotor, before 
deformation, is shown on the left. The wake consists of a segment of the von Karman vortex street the main axis of which is parallel to the direction of the flow velocity $c_{1}$ in the stator reference frame. The wake vortices with positive (counterclockwise) rotation are marked green, while those of negative (clockwise) rotation - red. Moreover, small black dots which can be observed close to the vortices indicate positions which they would occupy when solely convected with the main flow, without vortex interactions with each other and flow boundaries. Large black circles on the right represent the rotor vortices. The stator wake approaches the rotor cascade flowing from the direction defined by the flow velocity $\mathrm{w}_{1}$ in the rotor reference frame. When inside the rotor passage, it deforms in such a way that two groups of vortices of positive and negative rotation sign, $+\Gamma_{\mathrm{S} 1}$ and $-\Gamma_{\mathrm{S} 1}$ respectively, can be clearly seen.

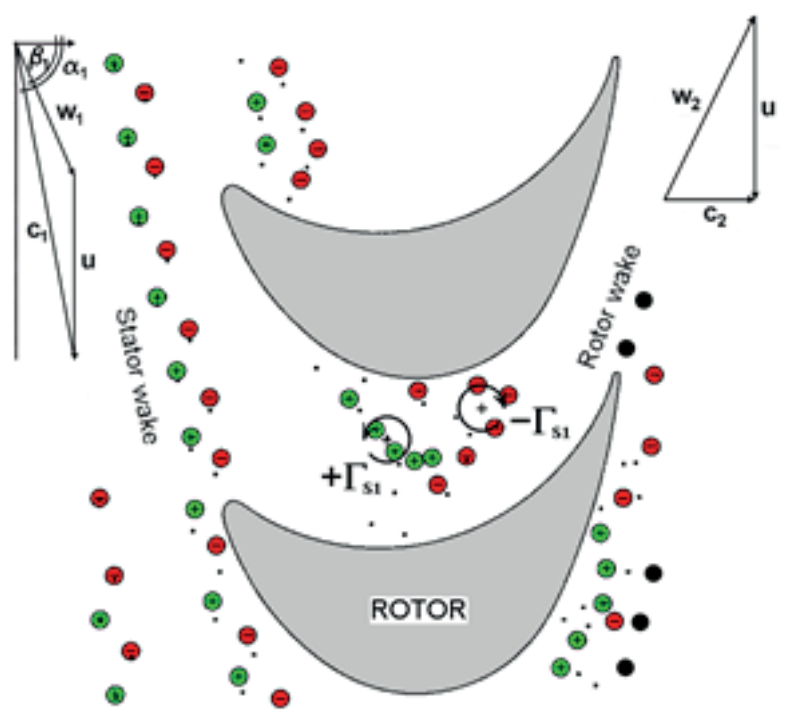

Fig. 3. Stator wake deformation inside rotor passage

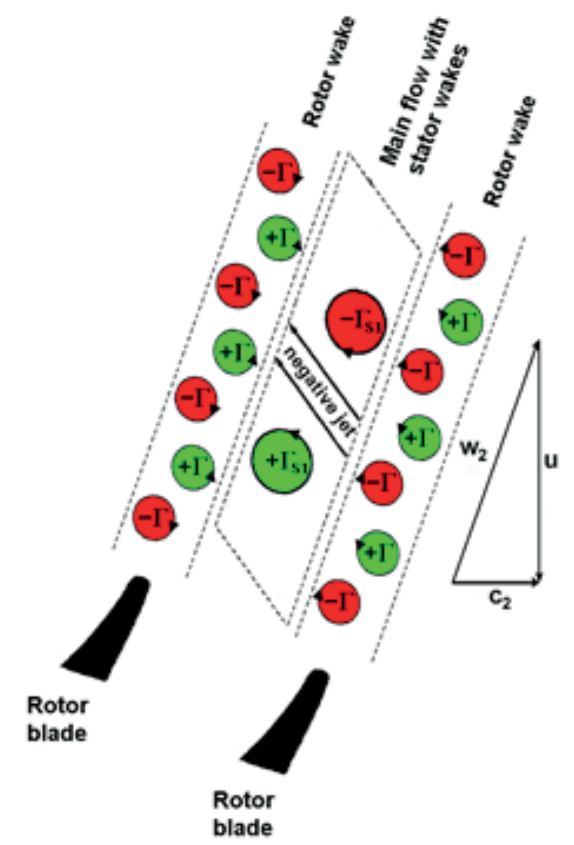

Fig. 5. Scheme of wake structure downstream of rotor $R$ cascade
Further behaviour of the stator and rotor wakes can be observed in Fig. 4 being a compilation of the results presented in [23-27]. All wakes leave the rotor moving, generally, in the direction of the main flow velocity $\mathrm{w}_{2}$. In this reference frame the stator vortices move one after another. However, in the reference frame connected with the $\mathrm{S}_{2}$ cascade their motion is controlled by the flow velocity component $c_{2}$ which is, as a rule, almost parallel to the turbine axis. As a consequence, in this reference frame the $S_{1}$ vortices move side by side like a vortex pair in the main $\mathrm{c}_{2}$ direction only slightly modified by their mutual interaction. In this context, the descriptions of the $\mathrm{S}_{1}$ wake motion which were mentioned at the beginning and which say that "the wake of the upstream stator impinges on the downstream stator leading edge", or "is convected through the middle of the downstream stator passage" seem to be rather imprecise, as configurations can be imagined in which one $S_{1}$ vortex impinges on the $S_{2}$ blade while the other flows through the middle of the $S_{2}$ passage, or both vortices flow inside one and the same the passage, or on opposite sides of one and the same blade. Figure 5 shows two extreme configurations in which the negative vortex flows through the passage and the positive vortex impinges on the blade leading edge (case a), or when the roles of the vortices are reversed (case b).
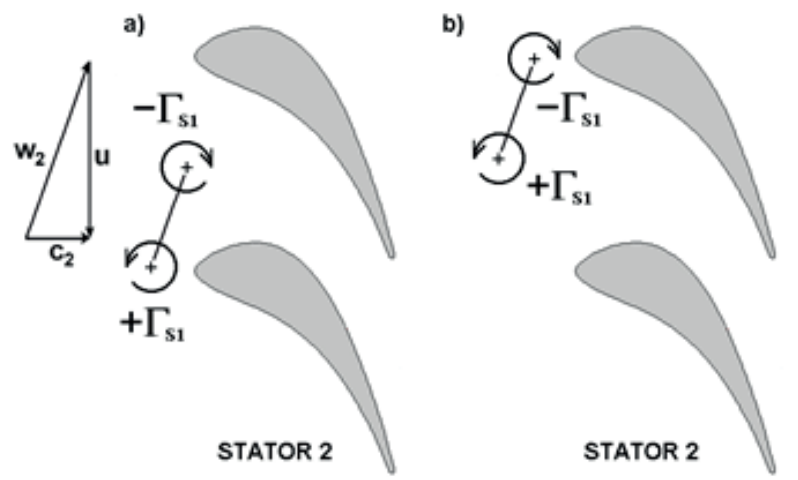

Fig. 5. Characteristic positions of $S_{1}$ wake vortices with respect to stator $S_{2}$ blades, where negative (a) or positive (b) vortex flows through $S_{12}$ passage.

Looking at the $S_{1}$ wake/ $S_{2}$ cascade interaction from the point of view of stator $S_{2}$ performance, the best situation is when the moving vortex increases the velocity on the suction side and/or decreases the velocity on the pressure side of the $\mathrm{S}_{2}$ blade, thus increasing the pressure differences between blade sides and the resultant lifting force. In the $\mathrm{S}_{2}$ cascade geometry shown in Fig 5 this conditions is met by the vortex $+\Gamma_{\mathrm{s} 1}$ while the other vortex $-\Gamma_{S 1}$ acts in the opposite way, i.e. unfavourably increases the velocity on the pressure side and decreases on the suction side of the stator $S_{2}$ blade. All this leads to the conclusion that the situation which is favourable for turbine performance is case $b$ ) when the negative vortex $-\Gamma_{S 1}$ is destroyed in the head-on collision with the $\mathrm{S}_{2}$ blade leading edge while the positive vortex $+\Gamma_{\mathrm{S} 1}$ moves relatively untouched through the centre of the $S_{2}$ passage, thus increasing the lift of both blades composing the passage. 


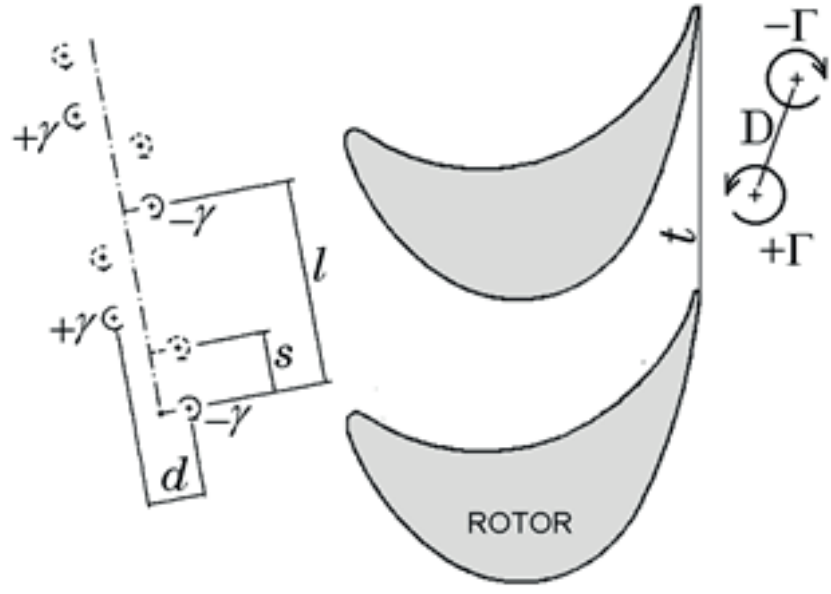

Fig. 6. Parameters of vortex structures upstream and downstream of rotor

The two vortex/blade configurations shown in Fig. 5 have been selected to illustrate basic features of vortex/blade interaction. As was already mentioned, a variety of other positions of the vortex pair $\left(-\Gamma_{\mathrm{S1}},+\Gamma_{\mathrm{S} 1}\right)$ with respect to the stator $\mathrm{S}_{2}$ blade are possible, depending on geometrical and flow parameters concerning the both stators and the rotor, see Fig. 6. The stator $\mathrm{S}_{1}$ geometry, especially the thickness and shape of the blade trailing edges and flow conditions in their vicinity, determine the strengths $+\gamma$ and $-\gamma$ of the $\mathrm{S}_{1}$ wake vortices and characteristic spatial distances of the von Karman street, which are: 1 - the distance between two vortices of the same rotation sign, and $\mathrm{d}$ - the distance between the opposite-sign rows. Of some importance for further interaction can also be the phase shift s of the entire von Karman structure with respect to the arbitrary basic position at the moment when the wake is cut off by the rotor blade [26]. The rotor geometry, in turn, decides upon the final strengths $+\Gamma_{S 1}$ and $-\Gamma_{S 1}$ of the $S_{1}$ wake vortices leaving the rotor and the distance $\mathrm{D}$ between them. For the same parameters of the wake approaching the rotor, the final parameters of the two vortices: $+\Gamma_{S 1},-\Gamma_{S 1}$, and $\mathrm{D}$, can be different depending on the pitch $t$ between the rotor blades, which controls the length of the wake segments cut off by consecutive rotor blades and, consequently, the number of vortices composing each segment. Also of some importance is the flow turning angle which may affect the course of the $S_{1}$ wake deformation inside the rotor $\mathrm{R}$ passage. Due to the abovementioned variety of parameters affecting the $S_{1}$ wake/ $S_{2}$ cascade interaction, its exact course and effects in a given turbine can only be obtained from CFD calculations performed for the geometry and flow conditions in this turbine.

When discussing clocking in real turbines it is also noteworthy to mention that the here presented analysis of stator wake behaviour bases on a two dimensional model which omits all three-dimensional effects connected, among other factors, with the presence of other vortex structures, such as horse-shoe and passage vortices for instance. Consequently, the above description of the course and effects of clocking can only be valid in those turbine regions in which the assumption about flow two-dimensionality is justified.
In turbine stages, especially those with radial blades, this region usually extends over a large central part of the passage, only excluding tip and hub areas where the activity of the three-dimensional vortices is dominating. In the abovementioned central part, a condition for the clocking to generate most remarkable and regular effects is parallel alignment of relevant pairs of blade edges: the $S_{1}$ trailing edge with the $\mathrm{R}$ leading edge, and the $\mathrm{R}$ trailing edge with the $\mathrm{S}_{2}$ leading edge, to provide opportunities for nearly twodimensional wake/blade interaction. It, however, does not mean that any clocking effects cannot be recorded in other turbine stage geometries. As the review of the literature shows, clocking effects can be recorded even in a turbine with an untypical stator-to-rotor blade count ratio $\mathrm{n}_{\mathrm{S}}: \mathrm{n}_{\mathrm{R}}=$ $=16: 96=1: 6$ in which the stator trailing edge is inclined to the radial rotor leading edge by as much as about 22 [deg] and, as a consequence, certain fragments of the wake shed from one stator blade pass, at the same time, through six consecutive rotor passages [28]. This result is explained by the fact that, irrelevant of the scale of complexity and threedimensionality of the flow, in a given clocking configuration the same fragments of flow structures leaving the rotor repeatedly impinge on and come into interaction with the same parts of the $\mathrm{S}_{2}$ blades. But, despite certain effects of clocking recorded, the information on this phenomenon gained from such untypical turbines is of little applicability in terms of extension to other geometries.

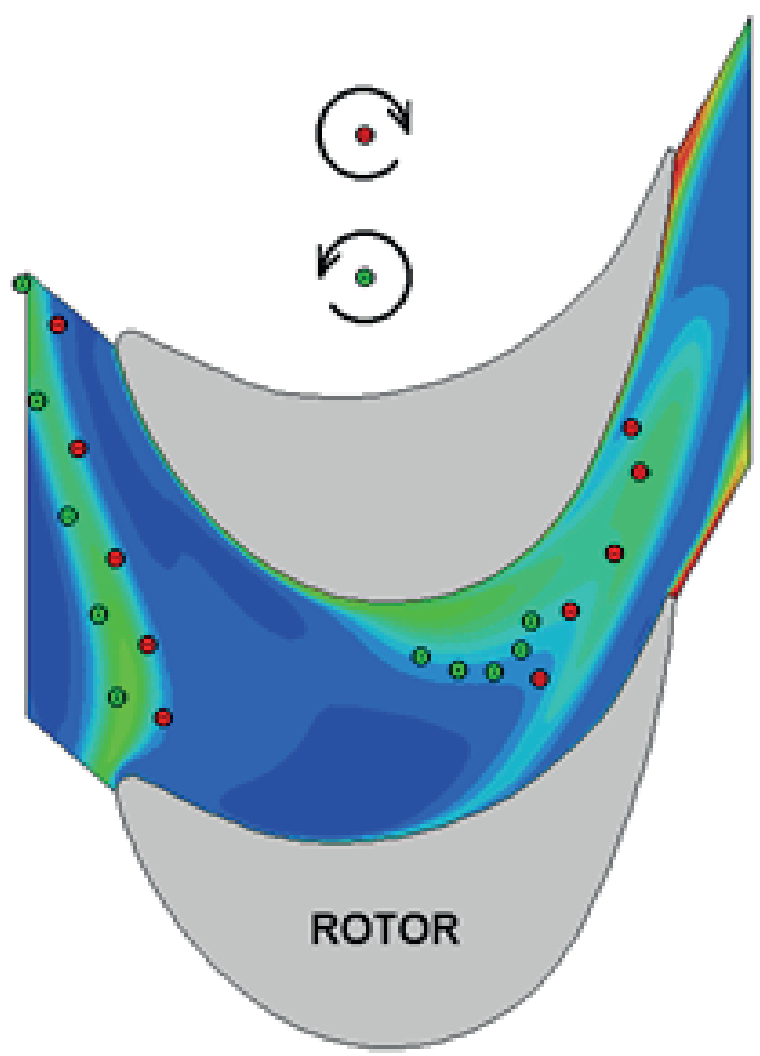

Fig. 7. Configuration of stator wake vortices inside rotor passage calculated using VDT against the entropy distribution obtained from CFD calculations. 
Finally, let us discuss the question whether there is any discrepancy between the earlier and presently formulated opinions about favourable and unfavourable positions of the $S_{1}$ wake with respect to $S_{2}$ blades from the point of view of clocking. Figure 7 shows an instantaneous distribution of $S_{1}$ wake vortices obtained from calculations based on Vortex Dynamics Theory on the background of the entropy distribution calculated by a CFD code solving NavierStokes equations complemented with a relevant turbulence model, here the k- $\omega$ SST model. We can notice that the area occupied by the negative (red) vortices coincides with the most distinctive front part of the CFD wake, while the positive (green) vortices do not have a clear representation in the CFD wake. When the wake has the form of a band of smooth velocity defect (or entropy increase, as in Fig. 2) without traces of coherent vortices, which is a standard pattern in clocking analyses based on CFD calculations, its deformed front part is frequently considered a representation of the entire wake and its impingement on the stator blade leading edge is believed to cover the full course of its interaction with the stator. In this context, the opinion on its head-on collision with the stator blade leading edge being the most favourable case of clocking is exactly the same as that formulated based on the vortex theory, which is only extended by including the presence of the other wake vortex $+\Gamma_{\mathrm{s} 1}$.

\section{Geometry conditions}

A commonly accepted general condition for clocking between two stators is the identity of their blade counts $n_{s 1}=n_{s 2}$. In this case all stator $S_{2}$ blades experience simultaneously the same clocking effects and the total result for the entire stator can be calculated by multiplying the elementary effect experienced by a single blade by the number of blades. Let us analyse now what happens when the condition $\mathrm{n}_{\mathrm{s} 1}=\mathrm{n}_{\mathrm{s} 2}$ is not strictly met.

$$
\text { a) } n_{S 2}<n_{S 1}
$$

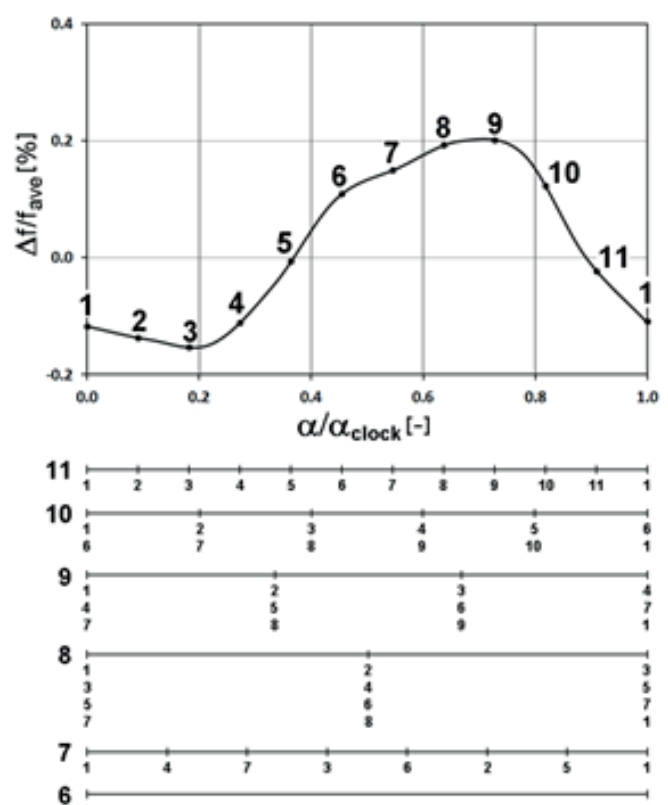

b) $n S 2>n S 1$
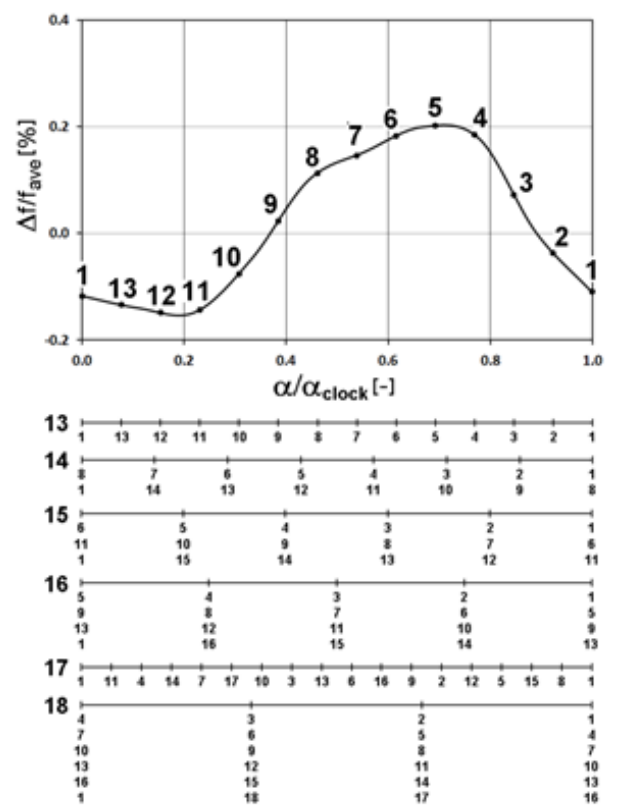

Fig 8. Clocking potential for $n_{S 1} \neq n_{S 2}$

For these studies let us employ a curve shown in Fig 8 which presents a hypothetical distribution of changes of an arbitrary parameter $f$ experienced at a given time by a single $\mathrm{S}_{2}$ blade after rotating it by the assumed clocking angle $\alpha$. The rotation range covers one interval aclock of clocking repeatability and all $\mathrm{f}$ values refer to one instantaneous time $\tau$ of rotor rotation, i.e. one instantaneous configuration of stator $\mathrm{S}_{1} /$ rotor / stator $\mathrm{S}_{2}$ cascades (To avoid misinterpretation it is necessary to stress here that the curve in Fig. 8 is not similar to typical clocking characteristics which usually present time-histories of parameter changes recorded individually for each clocking angle. In this sense the presented curve represents sort of cross-section of a set of those characteristics for a given time, and will change for different times.). Let us assume that this curve was prepared for a turbine with stator blade counts $\mathrm{n}_{\mathrm{S} 1}=\mathrm{n}_{\mathrm{S} 2}=12$, i.e. for a classical case of clocking geometry. As already mentioned, in this case each point of the curve, point 1 for instance, represents the clocking effect $f$ experienced not only by a single blade of concern, but also by all remaining $\mathrm{S}_{2}$ blades. The resultant total clocking effect for the entire cascade $S_{2}$ at time $\tau$ can be calculated by multiplying the current value of $f$ by the number of blades. The clocking repeatability interval $\alpha_{\text {clock }}$ is in this case equal to the angle $\alpha_{t}$ of $S_{2}$ rotation by one pitch, here: $\alpha_{\text {clock }}=\alpha_{t}=2 \pi / 12$.

Now, let us imagine that we remove one blade from stator $\mathrm{S}_{2}$, thus creating the blade count ratio $\mathrm{n}_{\mathrm{S} 1}: \mathrm{n}_{\mathrm{S} 2}=12: 11$. In this case the blade pitch in $S_{2}$ becomes larger than that in $S_{1}$ and the clocking effect experienced by each $S_{2}$ blade at time $\tau$ is not the same anymore. For instance, when one of the blades, arbitrarily denoted as blade(1), is at the position $\alpha / \alpha_{\text {clock }}=0.0$, the next blade (2) takes the position marked 2 on the curve in Fig. 8, the blade (3) takes the position 3 - and so on. As a consequence, the total clocking effect for the entire cascade $\mathrm{S}_{2}$ is the sum of 11 different values read at 11 equidistant points on the curve. The angle $\alpha_{\mathrm{p}}$, describing the cascade part along 
which different clocking effects are observed on the blades, extends to $2 \pi$, i.e. the entire circumference. Consequently, all positive and negative effects of clocking observed at the same time cancel each other and their total effect on the entire stator $\mathrm{S}_{2}$ is close to zero.

Continuing the above procedure, let us remove another blade. In this case the blade count ratio is $\mathrm{n}_{\mathrm{S} 1}: \mathrm{n}_{\mathrm{S} 2}=12: 10$. A remarkable difference between this and the previous case is that now there are pairs of $\mathrm{S}_{2}$ blades which take the same position with respect to stator $S_{1}$, which are: blades 1 and 6, 2 and 7, 3 and 8,4 and 9, and 5 and 10. Consequently, the number of blades which experience different effects of clocking at the same time is twice as small as in the previous case, and the angle $\alpha_{p}$ of clocking effect extension is reduced to $\pi$. Further blade removals lead to 3 sections of 3 blades for a 9-bladed row, 4 sections of 2 blades for an 8-bladed row, 1 section of 7 blades, and finally 6 sections of 1 blade. These results are depicted schematically below the left-hand diagram in Fig. 8.

Now let us move in the opposite direction and increase the initial number of $S_{2}$ blades by one. This way the blade pitch in $\mathrm{S}_{2}$ becomes smaller than that of $\mathrm{S}_{1}$, and again, each blade will experience a different clocking effect at the same time $\tau$. This time, however, when we take the curve from Fig 8 as the reference pattern, the order of blades experiencing particular clocking effects is reversed, see the right-hand diagram. Further reasoning on adding extra blades to the stator $\mathrm{S}_{2}$ is similar to that when they were removed. The resultant numbers of blades experiencing the same clocking effect when $\mathrm{n}_{\mathrm{s} 2}$ is increased from 12 to 18 are shown in Fig 8 below the right-hand diagram.

In this context a question can be raised whether any changes of operating parameters of the turbine can be obtained by clocking when $\mathrm{n}_{\mathrm{S} 1} \neq \mathrm{n}_{\mathrm{S} 2}$. In fact they can, but their real range, measured as the interval $\alpha_{\text {clock }}$ of effective $S_{2}$ clocking, is very limited. When $\operatorname{abs}\left(\mathrm{n}_{\mathrm{s} 1}-\mathrm{n}_{\mathrm{S} 2}\right)=1$ and there are no repetitions in relative $S_{1} / S_{2}$ blade configurations, the clocking interval $a_{\text {clock }}$ simply corresponds to the difference between the blade pitches in the clocked rows, as the rotation by an angle larger than $\alpha_{\text {clock }}$ will be a repetition of a blade configuration from within this range, only with another blade taking the "basic" position.

When the blade counts provide opportunities for a number of $S_{2}$ blades to take the same position with respect to the $S_{1}$ cascade, this decreases the number of blades experiencing different clocking effects and the resultant clocking interval is larger, as obtained by multiplying the basic value by the number of repetitions.

Without pure mathematical proof, the following general formula can be proposed for the clocking interval $a_{\text {clock }}$ of two arbitrary rows with blade counts $\mathrm{n}_{\mathrm{S} 1}$ and $\mathrm{n}_{\mathrm{S} 2}$ :

$$
\alpha_{\text {clock }}=\frac{2 \pi}{n_{S 1} n_{S 2}} \operatorname{gcd}\left(n_{S 1}, n_{S 2}\right)
$$

In this formula the fraction $2 \pi /\left(\mathrm{n}_{\mathrm{S} 1}{ }^{*} \mathrm{n}_{\mathrm{S} 2}\right)$ represents the basic value, which is true for cascades with arbitrary blade counts without common divisors, and $\operatorname{gcd}\left(\mathrm{n}_{\mathrm{s} 1}, \mathrm{n}_{\mathrm{s} 2}\right)$ is the greatest common divisor of blade counts $n_{\mathrm{S} 1}$ and $n_{\mathrm{S} 2}$ which takes into account repetitions of identical relative blade positions.

The values of characteristic clocking parameters for cases $\mathrm{n}_{\mathrm{S} 1} \neq \mathrm{n}_{\mathrm{S} 2}$ and $\mathrm{n}_{\mathrm{S} 2}$ changing from 6 to 18 are given in Tab. 1 . New symbols $\mathrm{n}_{\mathrm{S} 2 \mathrm{R}}$ and $\mathrm{n}_{\mathrm{S} 2 \mathrm{~S}}$ in the table represent the number of $S_{2}$ blades which occupy the same position with respect to stator $S_{1}$ and the number of different-value $S_{2}$ blades in one sequence. In particular, $\mathrm{n}_{\mathrm{S} 2 \mathrm{R}}=1$ means that different clocking effects are recorded on all $\mathrm{S}_{2}$ blades, along the entire row circumference, while $\mathrm{n}_{\mathrm{S} 2 \mathrm{~S}}=1$ means that each $\mathrm{S}_{2}$ blade experiences the same clocking effect.

Tab. 1. Characteristic clocking parameters for cases: $n_{S 1} \neq n_{S 2}$

\begin{tabular}{|c|c|c|c|c|c|}
\hline$n_{S 1}$ & $n_{S 2}$ & $\alpha_{p}$ & $n_{S 2 R}$ & $n_{S 2 S}$ & $\alpha_{\text {clock }}$ \\
\hline 12 & 12 & - & 12 & 1 & $2 \pi$ \\
\hline 12 & 11 & $2 \pi$ & 1 & 11 & $\frac{1}{11} * \frac{2 \pi}{3}$ \\
\hline 12 & 10 & $\Pi$ & 2 & 5 & $\frac{2}{10} * \frac{2 \pi}{-}$ \\
\hline 12 & 9 & $2 \pi / 3$ & 3 & 3 & $\frac{3}{9} * \frac{2 \pi}{}$ \\
\hline 12 & 8 & $\pi / 2$ & 4 & 2 & $\frac{4}{8} * \frac{2 \pi}{2}$ \\
\hline 12 & 7 & $2 \pi$ & 1 & 7 & $\frac{1}{7} * \frac{2 \pi}{}$ \\
\hline 12 & 6 & $\pi / 3$ & 6 & 1 & $\frac{6}{6} * \frac{2 \pi}{-}$ \\
\hline 12 & 13 & $2 \pi$ & 1 & 13 & $\frac{1}{13} * \frac{2 \pi}{-}$ \\
\hline 12 & 14 & $\pi$ & 2 & 7 & $\frac{2}{14} * \frac{2 \pi}{3}$ \\
\hline 12 & 15 & $2 \pi / 3$ & 3 & 5 & $\frac{3}{15} * \frac{2 \pi}{-}$ \\
\hline 12 & 16 & $\pi / 2$ & 4 & 4 & $\frac{4}{16} * \frac{2 \pi}{4}$ \\
\hline 12 & 17 & $2 \pi$ & 1 & 17 & $\frac{1}{17} * \frac{2 \pi}{2}$ \\
\hline 12 & 18 & $\pi / 3$ & 6 & 3 & $\frac{6}{18} * \frac{2 \pi}{2}$ \\
\hline
\end{tabular}

We can see from formula 1 that the narrowest opportunities for clocking are offered by two cascades with blade counts without common divisors, and in this case $\alpha_{\text {clock }}$ is the result of pure division of the full angle $2 \pi$ by the product of the two blade counts. The existence of common blade count divisors provides opportunities for increasing the clocking interval, and for the most extreme case, when $\mathrm{n}_{\mathrm{S} 1}=\mathrm{n}_{\mathrm{s} 2}$, it leads to the classical case of clocking in which $\alpha_{\text {clock }}$ is equivalent to $\alpha_{t}$. In this sense, to justify the opinion quoted at the beginning of this chapter: the case when the blade counts of the clocked rows differ by one can be considered one of worst cases for 
clocking, as in this case the blade counts usually do not have common divisors.

For arbitrary pairs of cascades $S_{1}$ and $S_{2}$, especially those without common divisors, the interval $\alpha_{\text {clock }}$ of effective clocking is a small fraction of the angle $\alpha_{t}$. In this context, taking into account that clocking experiments are usually preformed within the angle interval equal to $\alpha_{t}$ and not to $\alpha_{\text {clock }}$, a question should be raised whether rotating the $S_{2}$ cascade by a larger angle than $\alpha_{\text {clock }}$ will provide any clocking effects. The answer is positive, but certain difficulties can occur when interpreting the obtained results. To make this more clear let us illustrate the problem based on the initially analysed classical case of clocking, when $n_{\mathrm{s} 1}=n_{\mathrm{s} 2}=12$. For this case $\alpha_{\text {clock }}=\alpha_{t}$ and, what is obvious, rotating the $S_{2}$ cascade by an arbitrary small angle $\alpha_{c}$ within the range $\left(0, \alpha_{t}\right)$, will produce a curve similar to that shown in Fig. 8. But the same curve will be obtained when we rotate repeatedly the cascade by the angle $\alpha_{t}+\alpha_{c}$, or more generally: $\alpha_{t}+n^{*} \alpha_{c}$ where $n$ is an arbitrary positive integer, and the only difference is that we record the values in different cascade passages. What is more, when we rotate the cascade by $a_{t}-a_{c}$, we obtain the same curve in qualitative and quantitative terms, but with the sequence of points ordered in the opposite direction. A most complicated and most difficult for interpretation case would be when the cascade was repeatedly rotated by an angle $\alpha_{t}+\alpha_{c}$ and the angle $\alpha_{c}$ was close to $0.5^{*} \alpha_{t}$. The sequence of points obtained for this case would be accidental and their real position on the curve could only be found by subtracting full multiples of $\alpha_{t}$ for each point individually to reduce it to the basic interval $\left(0, a_{t}\right)$.

The same effect will be observed when recording the effects of clocking for cascades with $\mathrm{n}_{\mathrm{s} 1} \neq \mathrm{n}_{\mathrm{S} 2}$. But unlikely the classical case in which the angle $\alpha_{\text {clock }}=\alpha_{t}$ has a clear physical representation which can be easily used to assess the interval to which a given point belongs and to reduce it to the basic interval $\left(0, a_{\mathrm{t}}\right)$, for $\mathrm{n}_{\mathrm{S} 1} \neq \mathrm{n}_{\mathrm{S} 2}$ the need for reducing the results to the basic interval $\left(0, a_{\text {clock }}\right)$ is not so evident. But without doing this we can obtain a curve with an accidentally ordered sequence of points.

\section{Conclusions}

The article discusses two aspects of clocking in turbines with the aim to check the accuracy of commonly accepted opinions concerning the physical nature of clocking and geometric parameters of turbine stages which are believed to be more or less favourable for this phenomenon. The first opinion says that most favourable case of stator clocking in the turbine is when the wake of the upstream stator impinges on the downstream stator leading edge, while in the least favourable case the wake flows through the middle of the stator passage. The vortex dynamics theory based analysis has proved that the above opinion may refer to the negative vortex $-\Gamma_{\mathrm{S1}}$, one of two vortices created from the stator $S_{1}$ wake during its interaction with the rotor cascade. The other vortex, $+\Gamma_{\mathrm{S1}}$, when passing through the $2^{\text {nd }}$ stator passage generates positive flow disturbances and its possible destabilisation after head-on collision with the stator blade leading edge would be unfavourable for turbine performance.
The second opinion says that highest performance improvement can be obtained in turbines in which blade count ratios of the clocked rows is close to one, and when it is far from unity the clocking effect can be negligible. The here presented analysis of instantaneous distributions of flow parameters affected by clocking in turbines in which $\mathrm{n}_{\mathrm{S} 1} \neq \mathrm{n}_{\mathrm{S} 2}$ has shown that the highest angular range of clocking is obtained when $\mathrm{n}_{\mathrm{S} 1}$ is exactly the same as $\mathrm{n}_{\mathrm{S} 2}$, while the case when they slightly differ, by one for instance, can be considered one of the worst cases from the point of view of turbine performance improvement. A heuristic formula to calculate the range of the effective clocking interval $\alpha_{\text {clock }}$ for turbines with arbitrary blade count ratios of the clocked rows is proposed.

\section{References:}

1. Huber, F. W., Johnson, P. D., Sharma, O. P., Staubach, J. B., Gaddis, S. W., 1996, "Performance Improvement Through Indexing of Turbine Airfoils. Part 1: Experimental Investigation", ASME J. Turbomach., Vol. 118, pp. 630-635.

2. Griffin L. W., Huber F. W., Sharma O. P., 1996, "Performance Improvement Through Indexing of Turbine Airfoils. Part 2: Numerical Simulation”. ASME J. Turbomach., Vol. 118, pp. 636-642

3. Eulitz, F, Engel, K, Gebbing, H., 1996, "Numerical Investigation of the Clocking Effects in a Multistage Turbine, ASME Paper 96-GT-26, 1996

4. Dorney, D., Sharma, O. P.,1996, "A Study of Turbine Performance Increases Through Airfoil Clocking," AIAA Paper 96-2816, 1996

5. Cizmas P.G.A., Dorney D., 2000, “The Influence of Clocking on Unsteady Forces of Compressor and Turbine Blades", International Journal of Turbo and Jet Engines, Vol. 17, No. 2, pp. 133-142

6. Dorney, D. J., Croft, R. R., Sondak, D. L., Stang, U. E., Twardochleb, C. Z., 2001, "Computational study of clocking and embedded stage in a 4-stage industrial turbine", ASME Paper 2001-GT-509

7. Reinmoller U., Stephan B., Schmidt S., Niehuis R., 2002, "Clocking Effects in a 1.5 Stage Axial Turbine - Steady and Unsteady Experimental Investigations Supported by Numerical Simulations", ASME Journal of Turbomachinery, Vol. 124, No. 1, pp. 52-60

8. Cizmas P.G.A., Dorney D., 1999, "Parallel Computation of Turbine Blade Clocking”, International Journal of Turbo and Jet Engines. Vol. 16, No. 1, pp. 49-60

9. Li Wei, Zhu Xiao-cheng, Ouyang Hua, Du Zhao-hui, 2012, "Numerical Simulation of Clocking Effect on Blade Unsteady Aerodynamic Force in Axial Turbine", Journal of Thermal Science, Vol. 21, No. 5, pp 474-482 
10. Arnone A., Marconcini M., Pacciani R., 2000, “On the Use of Unsteady Methods in Predicting Stage Aerodynamics Performance", Proc International Symposium on Unsteady Aerodynamics, Aeroacoustics, and Aeroelasticity of Turbomachines ISUAAAT, Lyon, France, pp.24-36

11. Arnone A., Marconcini M., Pacciani R., Schipani C., Spano E., 2002, "Numerical Investigation of Airfoil Clocking in a Three-Stage Low-Pressure Turbine", Journal of Turbomachinery, Vol. 124, pp 61-68

12. Arnone, A., Marconcini, M., Greco, A., Spano, E., 2004, "Numerical Investigation of Three-Dimensional Clocking Effects in a Low Pressure Turbine", ASME Journal of Turbomachinery, Vol. 126, pp. 375-384

13. Meyer, R. X., 1958, “The Effect of Wakes on the Transient Pressure and Velocity Distributions in Turbomachines," ASME Journal of Basic Engineering, Vol. 80, pp. 15441552

14. Smith, L. H., 1966, "Wake Dispersion in Turbomachines," ASME Journal of Basic Engineering, Vol. 88, September, pp. 688-690

15. Lienhart, W., 1974, "Berechnung der instationären Strömung durch gegeneinander bewegte Schaufelgitter und der Schaufelkraftschwankungen", VDI-Forschungsheft, 562, VDI Verlag, Düsseldorf

16. Krammer, P., 1982, “Computation of Unsteady Blade Forces in Turbomachines by Means of Potential Flow Theory and by Simulating Viscous Wakes", ASME Paper 82-GT-198

17. Sieverding, C. H., Heinemann, H., 1990, “The Influence of Boundary Layer State on Vortex Shedding From Flat Plates and Turbine Cascades", ASME Journal of Turbomachinery, vol. 112, pp. 181-187

18. Cicatelli, G., Sieverding, C. H., 1997, “The Effect of Vortex Shedding on the Unsteady Pressure Distribution Around the Trailing Edge of a Turbine Blade," ASME Journal of Turbomachinery, Vol. 119, pp. 810-819

19. Mensink, C., 1996, "Numerical Prediction of Periodic Vortex Shedding in Subsonic and Transonic Turbine Cascade Flows", International Journal for Numerical Methods in Fluids, Vol. 22, pp. 881-897

20. Currie, T. C., Carscallen, W. E., 1998, "Simulation of Trailing Edge Vortex Shedding in a Transonic Turbine Cascade," ASME Journal of Turbomachinery, Vol. 120, pp. $10-19$

21. Arnone, A., Marconcini, M., Pacciani, R., 1999, "On the Use of Dual Time Stepping in Unsteady Turbomachinery Flow Calculations," ERCOFTAC Bulletin No. 42, September, pp. 37-42
22. Magagnato, F., 1999, "Unsteady flow past a turbine blade using non-linear two-equation turbulence models," Proceedings III European Conference on Turbomachinery: Fluid Dynamics and Thermodynamics, London, March, pp. 221-230

23. Kost, F., Hummel, F., Tiedemann, M., 2000, "Investigation of the Unsteady Rotor Flow Field in a Single HP Turbine Stage," Proceedings ASME TURBO EXPO 2000, May 8-11, Munich, Germany, Paper 200-GT-432, pp. 1-11

24. Tiedemann M., Kost F., 2001, "Some aspects of WakeWake Interactions Regarding Turbine Stator Clocking", ASME J. Turbomachinery, Vol. 123, pp.526-533

25. Hummel, F.,2001, "Wake-Wake Interactions and Its Potential for Clocking in Transonic High Pressure Turbine," Proceedings ASME TURBO EXPO 2001, June 4-7, New Orleans, U.S.A, Paper 2001-GT-0302, pp. 1-9

26. Swirydczuk J., 2002, "Vortex Dynamics of the Stator WakeRotor Cascade Interaction”, ASME J. Fluids Engineering, Vol. 124, pp. 400-412

27. Swirydczuk J., 2013, "Wake-blade interaction in steam turbine stages", Polish Maritime Research, Vol. 20, No. 2 (78), pp. 30-40

28. Krysinski J.E., Blaszczak J. R., Smolny A., 2005, "Stator Clocking Effects on 3D Flow in a Two-Stage Low-Pressure Turbine", Proc ASME TURBO EXPO 2005, Power for Land, Sea and Air, June 6-9, 2005, Reno-Tahoe, Nevada, USA, Paper GT2005-68811, pp. 1-12

\section{CONTACT WITH AUTHOR}

$$
\text { Jerzy Swirydczuk }
$$

Institute of Fluid-Flow Machinery

Polish Academy of Science

14 J. Fiszera St

80-231 Gdańsk

Poland

jerzy.swirydczuk@imp.gda.pl 